\title{
Poster Abstract: Integrating Multiple Sensor Modalities for Environmental Monitoring of Marine Locations
}

\author{
Edel O'Connor, Alan F. Smeaton, Noel E. O'Connor and Dermot Diamond \\ edel.oconnor@computing.dcu.ie \\ \{Alan.Smeaton,Noel.OConnor,Dermot.Diamond\}@dcu.ie \\ CLARITY: Centre for Sensor Web Technologies \\ Dublin City University, Ireland
}

\begin{abstract}
In this paper we present preliminary work on integrating visual sensing with the more traditional sensing modalities for marine locations. We have deployed visual sensing at one of the Smart Coast WSN sites in Ireland and have built a software platform for gathering and synchronizing all sensed data. We describe how the analysis of a range of different sensor modalities can reinforce readings from a given noisy, unreliable sensor.
\end{abstract}

\section{Categories and Subject Descriptors}

H.3.3 [Information Search \& Retrieval]: Information filtering; H.5.2 [User Interfaces]: Miscellaneous; I.4.8 [Scene Analysis]: Color, object recognition, sensor fusion, time varying imagery

\section{General Terms}

Measurement, Design, Experimentation

\section{Keywords}

Visual Sensing, Sensor Networks, Data Visualization

\section{INTRODUCTION}

Visual sensing is now ubiquitous and with the falling cost in hardware, it is now feasible to build visual sensor networks along the same line as other sensor networks. There are a variety of applications for these in environmental monitoring. Examples include monitoring features of a coastal location such as beach erosion or monitoring water quality. Coastal video systems have been identified as effective tools for coastal monitoring. A prime example of this is the major European research project entitled CoastView [1]. This work focused on the development of video systems in support of coastal zone management utilizing Argus technology [2]. However, little work has been carried out which utilizes visual sensor networks for broader environmental monitoring. Also, when monitoring a coastal location, it may be useful to use multiple sources of evidence in a manner such that gaps or errors in the sensing from some devices caused by, for example, mis-calibration, can be compensated for by

Copyright is held by the author/owner(s).

SenSys'08, November 5-7, 2008, Raleigh, North Carolina, USA.

ACM 978-1-59593-990-6/08/11. data from other devices. This also provides an opportunity to characterise the context of what is being sensed as using context ultimately facilitates better content analysis, indexing and access.

The overall objective of our work is to develop, test, deploy and then evaluate the effectiveness of a sensor network that includes visual sensing alongside more traditional sensor networks in order to monitor multiple aspects of the coastal marine environment. We are identifying coastal locations in Ireland where visual sensing, through cheap and easily deployed webcam-type CCTV devices, can be used to complement and enhance the usefulness of other sensors. The output is a generic software platform that gathers visual and other sensed information, automatically filters poor quality sensed images, carries out image analysis on retained visual data and links detected visual events to corresponding events detected from the sensor data. It will present a visualisation mechanism in the form of a summary tool for a domain expert to study linked features and events (e.g. looking at the various conditions under which an algal bloom occurs). One location has already been identified and preliminary work uses the data from this location. However, our work aims to build a highly adaptive system which could be transferable between locations and incorporate the various datasets relevant at the location in question, so we do not restrict ourselves in terms of data sources considered.

\section{DATA AGGREGATION}

Investigating marine locations for which there is multiple different sources of marine related environmental data available, is a continuous on-going process. However an initial test site was agreed upon for aggregation of data from multiple sensor sources. This location is situated at the Tyndall Institute in Cork, Ireland, which lies on the banks of the River Lee. This urban site is an attractive choice since the SmartCoast multi-sensor system for water quality monitoring is already deployed at this location [3].

\subsection{Data Sources}

A Wireless Sensor Network (WSN) deployed in the River Lee as part of the SmartCoast project enables in-situ chemical and physical measurements and allows the resulting data to be viewed in real time via the internet [3]. The key parameters being measured include water temperature, $\mathrm{pH}$, conductivity, water level, turbidity, light level, humidity and 


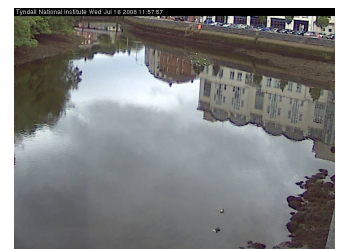

(a) Low water level

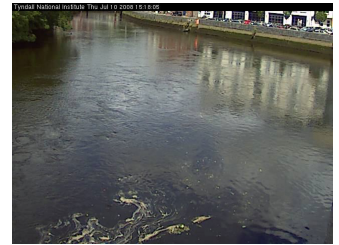

(b) Pollution

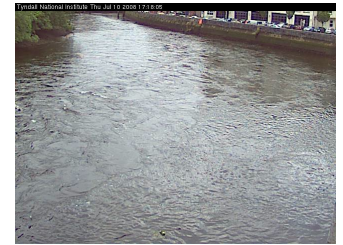

(c) Turbulence

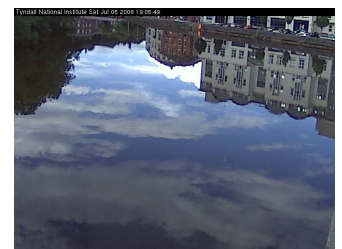

(d) Calm (lack of wind)

Figure 1: Environmental conditions during data capture

air temperature. The software application we have built retrieves this data every 15 minutes for further analysis.

An AXIS 212 PTZ Network camera was deployed at the same site as the in-situ sensors overlooking the River Lee. This camera is controlled remotely from a desktop PC at Dublin City University (DCU). It has a full 140 degree field of vision and whether in full overview mode or when zooming in, full 1:1 VGA resolution of $640 \times 480$ pixels is maintained. Images are automatically saved from the camera at four different angles at full zoom every ten minutes during daylight hours.

The Irish meteorological service provides an image with a $5 \mathrm{~km}$ resolution sampled every 30 minutes displaying rainfall radar data on their web site. The image from this web site is processed in order to determine clouds of rainfall along with their location, intensity, direction, and to predict their trajectory.

The use of satellite imagery can be considered to be another type of visual sensing, albeit perhaps more suited to coastal zones rather than inland waterways. However we consider satellite data in the context of this work to ensure that our system can support this type of data.

\subsection{Image Analysis}

Analysis needs to be carried out on the images in order to extract relevant data and to detect when an event is occurring. An event may constitute a change in the color of the water which may be due to algal growth, an increase in water level due to rainfall or opening of an upstream dam, the detection of an object floating on the water, an increase in the turbidity of the water, a weather event such as high wind or heavy rain etc. This is a difficult problem due to the difficulty in determining a background model for images where there may be changes in light intensity, reflections on the water, and rapid changes in the weather. However various techniques are being investigated some of which are based on those outlined in [4].

\subsection{Data Utilization and Visualization}

Methods for fusing these multiple datasets whereby data from an extensive sensor network can be viewed simultaneously and can enable experts to study various events and the conditions surrounding them, are being investigated. There are various approaches which can be undertaken here. However there are two aspects which need to be considered information retrieval and data visualization.

\section{PRELIMINARY INVESTIGATION}

Initial investigations have shown how the various datasets being utilized at the Tyndall site can greatly complement one another. Analysis of the dataset from the in-situ sen- sors has shown that there are occasionally gaps in the sensing whereby the sensors go offline or they are being cleaned. However these gaps can be partially compensated for with other sensing mechanisms. Image analysis techniques for example have been used to determine the water level, while the light values read from the in-situ sensors can be used in helping to determine image usefulness for visual data analysis. Visualization methods are also currently under investigation.

\section{FUTURE WORK}

Future work will involve fully developing a highly adaptive system which maximizes the opportunities that are generated from aggregating data from multiple sensors. There are many potential applications for this type of system. For example, biofouling is a huge problem in environmental sensing. This type of system could be used to detect and predict algal blooms which could be used in turn to determine when sensors need to be cleaned or when sites need to be managed.

\section{Acknowledgements}

Based on research funded by the Department of Communications, Marine and Natural Resources under the Strategy for Science, Technology and Innovation (2006-2013). Part of this work was funded by Science Foundation Ireland under grant $07 / \mathrm{CE} / \mathrm{I} 1147$.

\section{REFERENCES}

[1] M. Davidson, M. V. Koningsveldb, A. de Kruifc, J. Rawsond, R. Holmane, A. Lambertif, R. Medinag, A. Kroonh, and S. Aarninkhofb, "The coastview project: Developing video-derived coastal state indicators in support of coastal zone management," Coastal Engineering, no. 54, pp. 463-475, 2007.

[2] R. Holman and J. Stanley, "The history and technical capabilities of Argus," Coastal Engineering, no. 54, pp. 477-491, 2007.

[3] B. O'Flynn, R. Martinez-Catala, S. Harte, C. O'Mathuna, J. Cleary, C. Slater, F. Regan, D. Diamond, and H. Murphy, "Smartcoast: A wireless sensor network for water quality monitoring," in Second IEEE International Workshop on Practical Issues in Building Sensor Network Applications (SenseApp 2007), Sep 2007, pp. 1853-1856.

[4] P. KaewTraKulPong and R. Bowden, "An improved adaptive background mixture model for real-time tracking with shadow detection," in Second European Workshop on Advanced Video Based Surveillance Systems, Sep 2001. 